\title{
Video Article \\ Technique for Studying Arthropod and Microbial Communities within Tree Tissues
}

\author{
Nicholas C Aflitto ${ }^{1}$, Richard W Hofstetter ${ }^{1}$, Reagan McGuire ${ }^{1}$, David D Dunn ${ }^{2}$, Kristen A Potter ${ }^{1}$ \\ ${ }^{1}$ School of Forestry, Northern Arizona University \\ ${ }^{2}$ Acoustic Ecology Institute
}

Correspondence to: Richard W Hofstetter at rich.hofstetter@nau.edu

URL: https://www.jove.com/video/50793

DOI: doi: $10.3791 / 50793$

Keywords: Environmental Sciences, Issue 93, phloem sandwich, pine, bark beetles, mites, acoustics, phloem

Date Published: $11 / 16 / 2014$

Citation: Aflitto, N.C., Hofstetter, R.W., McGuire, R., Dunn, D.D., Potter, K.A. Technique for Studying Arthropod and Microbial Communities within Tree Tissues. J. Vis. Exp. (93), e50793, doi:10.3791/50793 (2014).

\section{Abstract}

Phloem tissues of pine are habitats for many thousands of organisms. Arthropods and microbes use phloem and cambium tissues to seek mates, lay eggs, rear young, feed, or hide from natural enemies or harsh environmental conditions outside of the tree. Organisms that persist within the phloem habitat are difficult to observe given their location under bark. We provide a technique to preserve intact phloem and prepare it for experimentation with invertebrates and microorganisms. The apparatus is called a 'phloem sandwich' and allows for the introduction and observation of arthropods, microbes, and other organisms. This technique has resulted in a better understanding of the feeding behaviors, lifehistory traits, reproduction, development, and interactions of organisms within tree phloem. The strengths of this technique include the use of inexpensive materials, variability in sandwich size, flexibility to re-open the sandwich or introduce multiple organisms through drilled holes, and the preservation and maintenance of phloem integrity. The phloem sandwich is an excellent educational tool for scientific discovery in both $\mathrm{K}$ - 12 science courses and university research laboratories.

\section{Video Link}

The video component of this article can be found at https://www.jove.com/video/50793/

\section{Introduction}

The phloem and bark tissues of conifers are host to thousands of organisms. Phloeophagy, the feeding on phloem tissues of the inner bark, is a habit usually associated with bark beetles, woodborers, and several other invertebrate and microbial taxa that live within trees. ${ }^{23}$ Bark beetles (Coleoptera: Curculionidae) develop and live within phloem except for short periods when adults seek new host trees. ${ }^{31}$ Bark beetles have been extensively studied due to their economic impacts on trees ${ }^{18,19}$, but direct behavioral observations of the insects within tree materials have been limited. ${ }^{4}$ Furthermore, the galleries constructed by bark beetles become habitat for a myriad of species. ${ }^{11}$ Vast numbers of fungi ${ }^{30}$, bacteria ${ }^{3}$, mites $^{10,21}$, and nematodes ${ }^{16,19}$ along with other predatory and parasitic arthropods ${ }^{22,24}$ inhabit the phloem material. The techniques provided here allow for direct observation of bark beetles, mites, and wood borers that typically live in subcortical environments. Slight alterations to the protocol can be made to study fungi and bacteria.

Bark beetles and associated organisms within tree tissues have been studied using a "phloem sandwich." Early use of this technique can be found in the literature dating back to 1933 , when it was used to observe the larval instars of Douglas-fir beetle (Dendroctonus pseudotsugae). ${ }^{2}$ The phloem sandwich has gone through many derivations as different materials became available. Originally, this device consisted of a piece of phloem placed between two plates of glass, pressed together by elastic bands. ${ }^{2}$ Later, clamps, tape, glue, plastic, and other materials have been used in the construction of the sandwich. ${ }^{3,14,15,17,26,28}$ The protocol described here offers improvements over some of the past designs. For example, in the past, test species were entered into the side of the sandwich, in between the plates of glass or plastic. This restricted the construction of galleries to one direction. The use of entry holes in the top plate allows greater freedom for test species to initiate natural gallery construction. Another advantage of the presented protocol is its simplified design, which can be easily constructed with few tools. The use of the phloem sandwich has allowed for direct observations of feeding behavior, reproduction, development, and interactions of organisms that would otherwise not have been possible. ${ }^{1,5,22}$ This method is also an excellent tool for K-12 education and science programs and displays.

There are several subtleties in the creation of a phloem sandwich that are difficult to interpret from or not reported in manuscripts. We believe that a visual (i.e., video) description of the production of a phloem sandwich is needed and would be of value to scientists and educators interested in studying phloeophagous organisms. Our protocol provides a simple and inexpensive way to observe arthropods, microbes, and other organisms that inhabit phloem tissues. 


\section{Phloem Selection and Removal from Tree}

1. Select a tree with particular characteristics. Collect phloem from pines (i.e., trees in the genus Pinus) as they have a distinctive phloem layer that is several millimeters thick. ${ }^{18,27}$ Once a tree is located that has few lower branches, inspect it for any other defects such as insect attacks and/or pathogens. Alternatively, use phloem from other conifer trees such as spruce trees in phloem sandwiches. ${ }^{9}$ Other trees species may be suitable for phloem removal such as hardwoods.

NOTE: Coniferous trees with large crowns typically have the thickest phloem tissue. To maximize the amount of phloem, it is best to cut trees that have few defects and branches on the trunk (bole). Pine species that are self-pruning work best. Phloem is typically thickest during the growing season and is thicker higher up the trunk than near the ground. Phloem is sometimes difficult to remove from trees during the fall or winter seasons.

2. Cut a tree down or use recently cut logs to obtain phloem tissue. Choose a falling direction that will minimize damage to the tree as well as nearby trees. Cut branches off the trunk to allow easier access to bark materials during phloem removal.

3. Once a tree is cut (felled; dropped on the ground) begin scraping the bark off the trunk with a sharp draw blade (Fig. 1A). Scrape an area of the bark off until the phloem layer is reached.

NOTE: The phloem layer is typically lighter in color (e.g., cream color) and moist, while the outer bark is drier and darker in color (Fig. 1B).

Take special care not to scrape the phloem. The area of bark scraped depends upon the size of the phloem piece(s) required.

4. After the bark is removed, cut an outline of the phloem piece with a sharp knife. Make sure the knife cuts all the way to the xylem (Fig. 2A).

5. To remove the phloem, start at a corner of the phloem piece using fingers to carefully peel back the phloem. Use a knife to assist in scraping the phloem off the xylem. Continue pulling the phloem off until the entire piece is removed.

NOTE: Phloem typically peels off of the tree easiest in the summer. For phloem that is exceedingly difficult to remove, a spatula-shaped too can aid in the effort.

6. Place the phloem piece immediately into a sterile bag. For best results, vacuum seal the bag (Fig. 2B) or, if using ziploc bags, remove all air from the bag. This increases the longevity of the phloem. Optionally, place multiple pieces of phloem in one bag. Store phloem in airtight bags slightly above freezing (between 1 and 10C) to retain its freshness.

\section{Creating the Phloem Sandwich}

1. Cut two equal pieces of clear acrylic, polycarbonate, or a similar hard, clear material (e.g., glass) slightly larger than the piece of phloem (Fig. 2C). Round the edges of the acrylic to prevent the corners from tearing the Parafilm seal. This protocol uses a 1/8 in. thick clear acrylic. NOTE: The size of the cut pieces depends on the needs of the subject organism and the length of the study. For instance, a pair of bark beetles utilizes $4 \mathrm{dm}^{2}$ of phloem over a month period, but will only need $1 \mathrm{dm}^{2}$ if the study takes place within several days.

2. Drill a hole into one of the acrylic pieces to allow entry of study organism(s). The size and number of holes depends on the objectives (Fig. 2D).

3. Prior to placing the phloem between the acrylic pieces, sterilize the acrylic surface (with $>70 \%$ ethanol) or, if using new acrylic, remove the protective film.

4. Place the fresh piece of phloem between the sterile acrylic pieces. Orient the acrylic piece with the hole(s) on the inner or outer side of the phloem, whichever is needed. Typically, face the outer side of the phloem (side that had bark on it) toward the entry holes.

\section{Sealing the Phloem Sandwich}

1. To create a temporary seal around the phloem sandwich, use 2 in. wide Parafilm strips pulled around the edges of the phloem sandwich (Fig. 2C). Alternately, use a polyvinylidene chloride wrap to seal the edges. ${ }^{6}$ Next, place a clamp on each side of the sandwich to squeeze the acrylic to the phloem. Make sure the entire surface is clamped down to prevent air space between the acrylic and phloem (Fig 2C). If not clamped properly, specimens may move in between the acrylic and phloem.

2. To create a semi-permanent seal add a non-gluing epoxy or petroleum jelly around the phloem. Make sure that the material completely surrounds the phloem. Next, use clamps or screws with bolts (may need to drill holes ahead of time) to hold the acrylic tight to the phloem. The sandwiches only remain viable for up to one or two months.

3. Depending on the oxygen needs of the study organisms, add an air filter on one or more sides of the sandwich. This will allow air to enter into the phloem sandwich but limit water loss from the phloem. We use simple charcoal filters that also reduce the risk of fungal and bacterial contamination.

4. For study specimens that require entry and exit at will, replace one of the acrylic pieces with wood or similar material that an insect can bore into. This is particularly important for the observation of wood borers, because after their larval state is completed in the phloem layer, they then bore into the xylem.

5. To prevent test subjects from exiting the entry holes, place small petri dishes (or other objects, tape) over the holes, blocking escape. Since organisms that reside in these spaces are accustom to low light levels, it may be necessary to place the sandwiches in a dark room or box, or place opaque material on top to block the light.

\section{Observing Organisms in the Phloem Sandwich}

1. Introduce study specimens into entry hole of the phloem sandwich (Fig. 2D). Observe specimens using a dissecting microscope under a red light or white light set at a low setting (Fig. 2E). 
2. To record the activities or growth of specimens within the phloem sandwich attach a camera or video camera to the microscope. Attach special video cameras to the microscope eyepiece (Fig. 2E \& F). For very small organisms such as mites, nematodes, and pseudoscorpions, use a high definition video camera attached to the microscope.

3. To record sounds, insert microphones into the entry hole of the phloem sandwich or through the side of the phloem sandwich. Because the entry hole is small, use a small microphone such as an electret condenser microphone.

4. To record from the side or surface of the phloem sandwich use a piezo element (Fig. 3B). To playback sound, use the same piezo element or attach a tactile transducer (excitor) to the surface of the top or bottom of the acrylic.

\section{Representative Results}

The protocol described above will enable a researcher to observe organisms that live in the cryptic environment underneath the bark of a tree. To illustrate the use of this technique we describe a representative study from our lab that uses this technique. ${ }^{12}$ In this experiment, phloem sandwiches were used to observe the effects of acoustic treatments on reproductive output, tunneling distance, and survival of bark beetles (Fig. 2E and 2F). Direct observation of beetles, made possible by the use of a phloem sandwich, revealed several interesting findings. First, we recorded daily tunneling distances by marking the beetles' location on the acrylic with a marker every $24 \mathrm{~h}$. This process revealed significant differences across sound treatments that would have been hidden without the phloem sandwich. Next, we observed a reduction in egg production with particular sound treatments. The mobile nature of the phloem sandwich allowed for egg observations to be completed under a dissecting microscope without disturbing the beetles. During these observations, we captured beetle egg laying behavior with a highdefinition camera attached to the microscope. Other notable observations included the killing of mates and the initiation of flight associated with particular acoustic treatments. The phloem sandwich assay was crucial to our study of bark beetle responses to acoustic treatments. These discoveries, which would be impossible to observe underneath the bark of a tree, are valuable contributions to developing management options for combatting bark beetle outbreaks. 


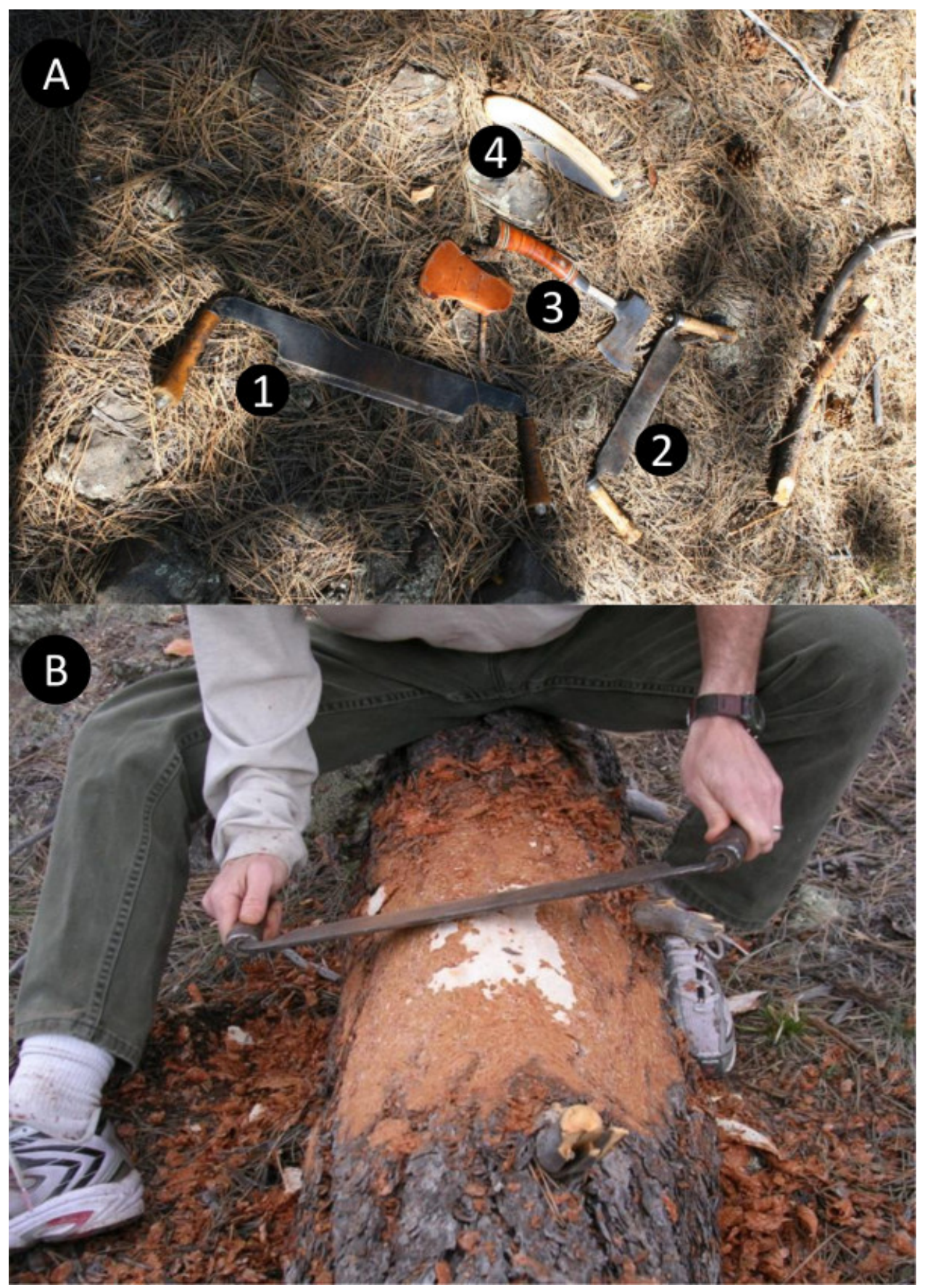

Figure 1. A) Tools needed to remove bark from tree. Items 1 and 2 are draw blades used to scrape bark off of the tree. Items 3 (hatchet) and 4 (pull saw) are useful to remove branches near shaving area. B) Use of draw blade to remove bark from tree. Notice the light color of the phloem underneath the reddish bark. 


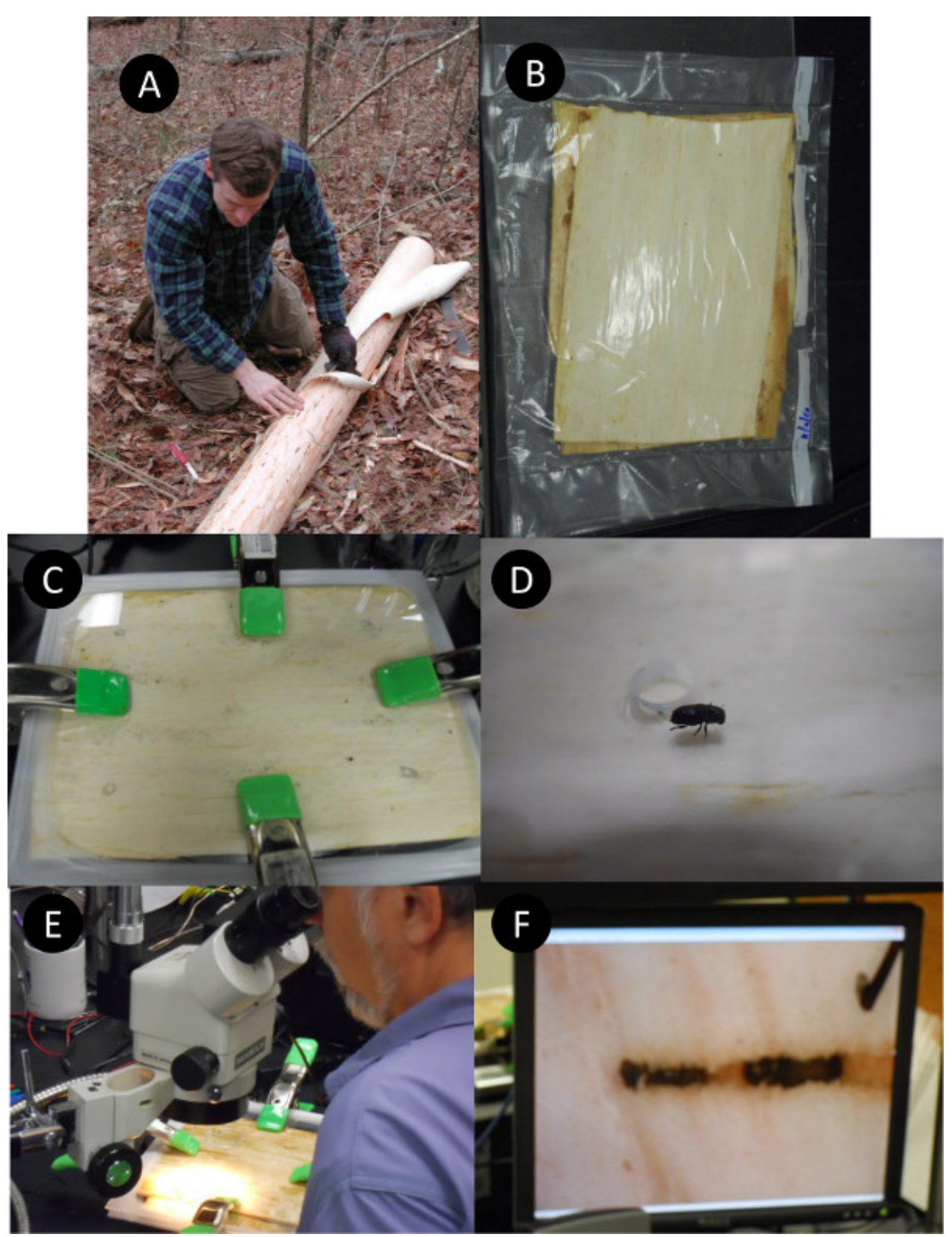

Figure 2. A) Removal of phloem after bark was scraped off of tree. B) Fresh phloem stored in vacuum sealed bag. C) Phloem sandwich with clamps holding the acrylic pieces together and Parafilm around edges to prevent contamination and desiccation of phloem. D) Bark beetle near hole drilled into acrylic . E) Using microscope to observe phloem sandwich. F) Video display of bark beetles within phloem sandwich. 


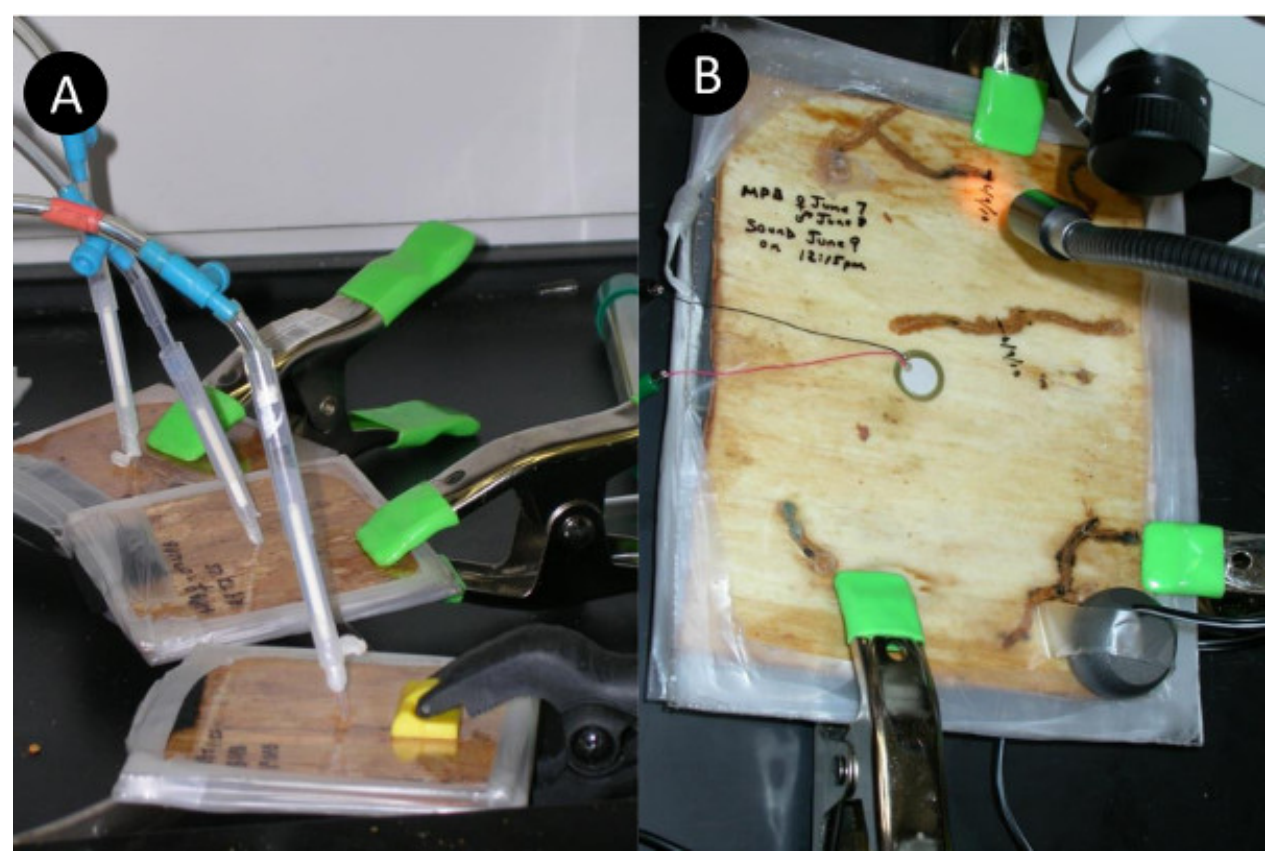

Figure 3. A) Pheromone acquisition from beetles in phloem sandwich. Air is being pulled through tubes containing Super-Q absorbent. B) Recording and playback of sound via a piezo-electric transducer (center of picture) to mountain pine beetles within phloem sandwich.

\section{Discussion}

The phloem sandwich allows for the introduction and observation of arthropods, microbes, and other small organisms that inhabit phloem tissues. $1,7,8,9,17,18$ This technique has resulted in new discoveries and a better understanding of behaviors, life-history traits, development, and interactions of organisms within tree phloem. ${ }^{1,5,10}$ The sandwich protocol described here is a hybrid of past designs, and will provide an economical sandwich that is easily constructed with minimal equipment and materials. The glass, acrylic, or polycarbonate pieces can be reused, and the only consumable materials are the Parafilm and phloem.

Although all steps in the protocol are important, certain steps should be followed strictly to ensure the highest degree of success. First, a tree should be located that has a portion of the bole free, or relatively free, of branches. Trees that have many branches are difficult to shave and will yield few viable phloem pieces, as every branch must be cut around when removing the phloem. Next, it is important to minimize air exposure of the phloem. We quickly place each phloem piece in a bag upon removal. Once three to six pieces are removed, they are transferred into a vacuum-sealed bag; we use a power inverter to run the sealer off a vehicle battery in the field. Last, sanitizing the acrylic pieces and general cleanliness when executing this protocol will reduce fungal growth in the sandwich. This is especially important for extended observations.

As mentioned above, there are limitations of the phloem sandwich apparatus. Since the phloem layer is thin, only small organisms that typically remain in the phloem can be introduced into the sandwich. Larger insects such as wood borers (i.e., Buprestid, Cerambycid species) can be introduced and observed for the early stages of their lifecycle. This timeframe is usually limited to two to three weeks; after this point the larvae require xylem wood to bore into for pupation. In contrast, for bark beetles, especially those in the genus Ips, a full lifecycle can be observed -including mating, egg hatching, feeding, pupating, and eclosion into an adult beetle. At this point, the viability of the sandwich is usually exhausted due to desiccation and fungal growth. ${ }^{28}$ Additionally, this apparatus does not allow insects to freely and naturally colonize or exit the phloem sandwich. ${ }^{27}$

Our protocol is flexible in terms of size, shape, and type of phloem used. Short studies require less phloem material and sandwich size can be scaled accordingly. Many coniferous species have been used as phloem donors in a phloem sandwich (e.g., ponderosa pine ${ }^{12}$, Douglas-fir ${ }^{2}$, spruce $^{29}$, loblolly pine ${ }^{27}$, longleaf pine ${ }^{27}$ ). Materials in the sandwich can also be altered; for example, glass plates can be used instead of acrylic and epoxy or tape instead of Parafilm.

The most difficult part of this protocol is the phloem removal process. Trees next to each other may differ in how difficult their phloem is to remove. When a tree has difficult phloem, patience is critical. In these cases, carefully run a knife between the hard xylem and the spongy phloem. This process feels literally like skinning the tree.

After mastering the basic sandwich technique, alterations to the protocol can help fit specific needs. For example, by honing the techniques required to remove phloem, larger pieces can be removed and used to create larger sandwiches. Also, alterations can be made to accommodate specific instruments, e.g., additional holes to monitor chemical emissions (Fig. 3A), or record or playback sounds (Fig. 3B). Modifications can be made to allow for semi-permanent preservation of the phloem and its organisms, or for temporary observations of organisms that can later be let free. 


\section{Disclosures}

No conflicts of interest declared

\section{Acknowledgements}

We thank Eli Jensen, Stefano Padilla, and Kasey Yturralde for assistance, and Karen London and anonymous reviewers of the manuscript. We thank Jake Baker and Karla Torres for video footage. Funding was provided to R.W.H by the NAU School of Forestry and NAU Technology and Research Initiative Fund (TRIF).

\section{References}

1. Aukema, B. H., Raffa, K. F. Behavior of adult and larval Platysoma cylindrical. (Coleoptera: Histeridae) and larval Medetera bistriata. (Diptera: Dolichopodidae) during subcortical predation of Ips pini. (Coleoptera: Scolytidae). J Insect Behav. 17, 115-128, doi: 0892-7553/04/0100-01115/0 (2004).

2. Bedard, W. D. The number of larval instars and the appropriate length of the larval stadia of Dendroctonus pseudotsugae. Hopk., with a method for their determination in relation to other bark beetle. J Econ Entomol. 26, 128-I 134 (1933).

3. Bridges, J. R. Nitrogen-fixing bacteria associated with bark beetles. Microb Ecol. 7, 131-137, doi:10.1007/BF02032495 (1981).

4. Cardoza, Y. J., Klepzig, K. D., Raffa, K. F. Bacteria in oral secretions of an endophytic insect inhibit antagonistic fungi. Ecol Entomol. 31, 636-635, doi:10.1111/j.1365-2311.2006.00829.x (2006).

5. Cardoza, Y. J., Moser, J. C., Klepzig, K. D., Raffa, K. F. Multipartite symbioses among fungi, mites, nematodes, and the spruce beetle, Dendroctonus rufipennis.. Environ Entomol. 37, 956-963, doi:10.1603/0046-225X(2008)37 [956:MSAFMN]2.0.CO;2 (2008).

6. Chen, H.-F., Salcedo, C., Sun, J.-H. Male mate choice by chemical cues leads to higher reproductive success in a bark beetle. Animal Behavior. 83, 421-427, doi:10.1016/j.anbehav.2011.11.012 (2012).

7. Dodds, K. J., Graber, C., Stephen, F. M. Facultative intra guild predation by larval Cerambycidae (Coleoptera) on bark beetle larvae (Coleoptera: Scolytidae). Environmental Entomology. 30, 17-22, doi: 10.1603/0046-225X-30.1.17 (2001).

8. Franklin, R. T. A technique for studying the insect parasites of Dendroctonus frontalis. and other bark beetles (Coleoptera: Scolytidae). Journal of Georgia Entomology Society. 2, 43-44 (1967).

9. Gries, G., Pierce Jr., H. D., Lindgren, B. S., Borden, J. H. New techniques for capturing and analyzing semiochemicals for Scolytid beetles (Coleoptera: Scolytidae). J Econ Entomol. 81, 1715-1720 (1988).

10. Hofstetter, R. W., Moser, J. C., McGuire, R. Observations of the mite Schizosthetus lyriformis. (Acari: Parasitidae) preying on bark beetle eggs and larvae. Entomology.News. 120, doi: 397-400, 10.3157/021.120.0408 (2009).

11. Hofstetter, R. W. Chapter 11: Mutualists and Phoronts of the Southern Pine Beetle. In. Southern Pine Beetle II (K.D. Klepzig \& R. Coulson eds.). United States Dept. of Agriculture Forest Service, Southern Research Station General Technical Report SRS-140. Pages 161-181 (2011).

12. Hofstetter, R. W., Dunn, D. D., McGuire, R. and Potter, K. A. Using acoustic technology to reduce bark beetle reproduction. Pest Manag Sci. 70, 24-27, doi:10.1002/ps.3656 (2014).

13. Hopping, G. R. Techniques for rearing Ips. De Geer (Coleoptera: Scolytidae). Can Entomol. 93, 1050-1063, doi:10.4039/Ent931050-11 (1961).

14. Hougardy, E., Gregoire, J-C. Cleptoparasitism increases the host finding ability of a polyphagous parasitoid species, Rhopalicus tutela. (Hymenoptera: Pteromalidae). Behav Ecol Sociobiol. 55, 184-189, doi:10.1007/s00265-003-0688-y (2003).

15. Kaston, B. J., Riggs, D. S. Studies on the larvae of the native elm bark beetle. J Econ Entomol. 30, 98-108 (1937).

16. Kinn, D. N. Life cycle of Dendrolaelaps neodisetus. (Mesostigmata: Digamasellidae), a nematophagous mite associated with pine bark beetles (Coleoptera: Scolytidae). Environ Entomol. 13, 1141-1144 (1984).

17. Kinn, D. N., Miller, M. C. A phloem sandwich unit for observing bark beetles, associated predators, and parasites. USDA FS Res. Notes SO-269. 3 pg (1981).

18. Lieutier, F., Day, K. R., Battisti, A., Gregoire, J-C., Evans, H. F. Bark and wood boring insects in living trees in Europe, a synthesis. Kluwer Academic Publishers, Boston USA. 569 pg (2004).

19. Massey, C. L. The influence of nematode parasites and associates on bark beetles in the United States. Bulletin of the Entomology Society of America. 12, 384-386 (1966).

20. Moser, J. C., Roton, L.M. Mites associated with southern pine bark beetles in Allen Parish, Louisiana. Can Entomol. 103, 1775-1798, doi: 10.4039/Ent1031775-12 (1971).

21. Mills, N. J. The natural enemies of scolytids infesting conifer bark in Europe in relation to the biological control of Dendroctonus. spp. in Canada. Biocontrol News Information. 4, 305-328 (1983).

22. Nagel, W. P., Fitzgerald, T. D. Medetera aldrichii. larval feeding behavior and prey consumption [Dipt.: Dolichopodidae]. Entomophaga. 20, 121-127, doi: 10.1007/BF02373458 (1975).

23. Paine, T., Raffa, K., Harrington, T. Interactions among scolytid bark beetles, their associated fungi, and live host conifers. Annu Rev Entomol. 42, 179-206, doi: 10.1146/annurev.ento.42.1.179 (1997).

24. Reeve, J. D. Predators of the southern pine beetle. In. Southern Pine Beetle II (Coulson, R. N.; Klepzig, K. D. eds). Gen. Tech. Rep. SRS-140. Asheville, NC: U.S. Department of Agriculture Forest Service, Southern Research Station. Pages 153-160 (2011).

25. Reid, R. W. The behavior of the mountain pine beetle, Dendroctonus monticolae. Hopkins, during mating, egg laying and gallery construction. Can Entomol. 90, 505-509, doi:10.4039/Ent90505-9 (1958).

26. Taylor, A. D., Hayes, J. L., Roton, L., Moser, J. C. A phloem sandwich allowing attack and colonization by bark beetles (Coleoptera: Scolytidae) and associates. J. Entomol. Soc. Amer. 27, 101-116 (1992).

27. Yu, C. C., Tsao, C.H. Gallery construction and sexual behavior in the southern pine beetle, Dendroctonus frontalis. Zimm. (Coleoptera: Scolytidae). Georgia Entomology Society. 2, 95-98 (1967). 
28. Yturralde, K. The Acoustic Ecology of Bark Beetles and Bed Bugs. PhD. Dissertation. Northern Arizona University. 323 pages (2013).

29. Wermelinger, B., Seifert, M. Analysis of the temperature dependent development of the spruce bark beetle Ips typographus. (L.) (Col., Scolytidae). Journal of Applied Entomology. 122, doi:185-191, 10.1111/j.1439-0418.1998.tb01482.x (1998).

30. Whitney, H. S. Relationships between bark beetles and symbiotic organisms. In. Bark Beetles in North American Conifers (J.B. Mitton and K.B. Sturgeon, eds.). University of Texas Press, Austin, TX. Pages 183-211 (1982).

31. Wood, S. L. The bark and ambrosia beetles of North and Central America (Coleoptera: Scolytidae), a taxonomic monograph. Brigham Young University, Provo, Utah. 1359 pgs (1982). 